\title{
Automated Rheumatic Heart Disease Detection from Phonocardiogram in Cardiology Ward
}

\author{
Melkamu Hunegnaw Asmare ${ }^{1,2,3}$, Frehiwot Woldehanna ${ }^{3}$, Luc Janssens ${ }^{1}$ and Bart Vanrumste ${ }^{1,2}$ \\ ${ }^{1}$ KU Leuven, Campus Group T, eMedia Research Lab, Leuven, Belgium \\ ${ }^{2}$ KU Leuven, Electrical Engineering Department (ESAT), STADIUS, Leuven, Belgium \\ ${ }^{3}$ Addis Ababa Institute of Technology, Center of Biomedical Engineering, Addis Ababa, Ethiopia \\ melkamhunegnaw.asmare@kuleuven.be
}

Keywords: Rheumatic Heart Disease, Machine Learning, Support Vector Machines, Phonocardiogram.

\begin{abstract}
Rheumatic Heart Disease (RHD) is a preventable and treatable form of cardiovascular diseases. It is also referred to as the ailment of the disadvantaged mainly affecting children and young adults. RHD is recognized as a global health priority by World Health Organization. This chronic heart condition silently deteriorates the normal function of the heart valves which can be detected as a heart murmur using a stethoscope. As the cardiac auscultation process is an elusive process, the clinician will always be tempted to refer the patient for expensive and sophisticated imaging procedures like echocardiography. In this study, a machine learning algorithm is developed to augment the limitation in the auscultation process and transform the stethoscope as a powerful screening tool. For this current study, an RHD heart sound data set is recorded from one hundred seventy subjects. A total of twenty-six features are extracted to model murmur due to RHD. Twenty-four classification and regression algorithms have been tested out of which the Cubic SVM has demonstrated superiority with a classification accuracy of $97.1 \%$, with $98 \%$ sensitivity, $95.3 \%$ of specificity $97.6 \%$ precision. The corresponding positive predictive values (PPV) are $96 \%$ and $97 \%$ for normal and RHD respectively. The results are based on data collected from a cardiology ward where there are more pathological cases than controls. Hence it is a valuable detection tool in a cardiology clinic. But in the future, integrating this machine learning algorithm with a mobile phone can be a powerful screening tool in places where access to echocardiography and cardiologist is difficult. Thus, it can then aid a timely, affordable and reliable detection tool allowing a non-medically trained individual to screen and detect RHD.
\end{abstract}

\section{INTRODUCTION}

Due to the rapid epidemiological transition observed in developing countries, not only communicable diseases but also noncommunicable diseases are becoming the major cause of death risks. Cardiovascular diseases, cancer, chronic respiratory disorders, and diabetes are the most common ones. Among these, cardiovascular disorder takes the leading role(WHOAnnualReport, 2013). Worldwide, ischemic heart disease is the number one cause of death, which affects males with age usually 65 or more (Emelia J. Benjamin, 2019). However, RHD is the leading cause of cardiovascular disorders in middle- and low-income countries. The average age is around 28 years with females affected twice as much as men (WatkinsDA, 2017).

RHD is caused by Group A streptococcal Bacteria (GAS) infection. These bacteria are normally found in the skin and in the throat of healthy people. GAS is an important cause of throat infection. In certain susceptible people, usually children, the immune system becomes confused and attacks both the GAS bacteria and parts of the host's body. This autoimmune at- tack causes the inflammation of the joints, skin, brain and most importantly the heart (Watkins DA, 2017). RHD is a chronic heart condition and early in the dis- ease, there are usually no symptoms. The disease can silently progress especially after repeated episodes of infection. Each episode brings renewed heart valve inflammation that eventually leads to local scarring and distortion of the valve architecture. First, the affected valve starts to leak, normally referred to as re- gurgitation; later the scarring can stop the valve from opening properly and make it narrow for sufficient blood passage, referred to as stenosis. These abnormalities create unusually turbulent blood flow in the heart chambers 
which is called heart murmur. Left untreated, RHD will compromise the cardiac output of the patient which will subsequently lead to pre- mature death (Walsh, 2019). The heart sound wave- form has distinct features called the first heart sound (S1), the second heart sound (S2), systole and diastole parts. Murmur normally presents itself in the systolic or diastolic parts. The heart sound can be listened to and recorded using a stethoscope in the form of a phonocardiograph (PCG). A three second MATLAB plot of clean, noisy and murmur types of heart sound is shown in Fig. 1. The clean signal is manually segmented to locate S1, S2, systole and diastole.

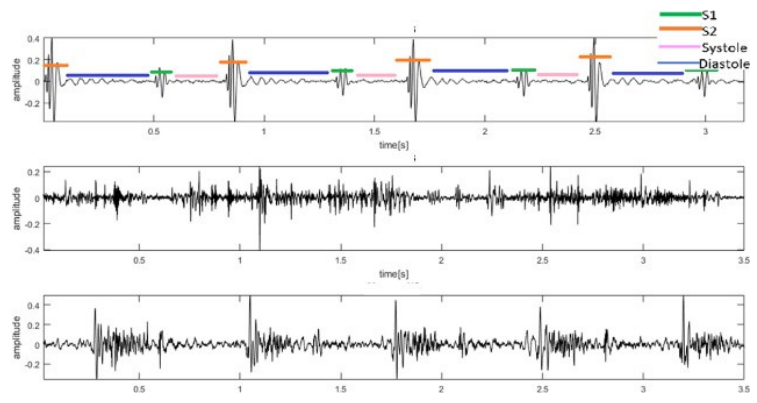

Figure 1: Time series representation of heart sounds: clean heart sound with S1, S2, Systole and Diastole labelled (top), noisy heart sound (middle), and heart sound with a murmur (bottom).

The heart sound gives vital information about cardiac wellbeing. However, even under ideal conditions, the accuracy of diagnosis is very low (Pelech, 2004). This is in reality attributed to the inherent limitation of the human auditory system to perform accurate auscultation. On top of that, the listening process is highly subjective. This usually forces doctors to be highly dependent on other expensive imaging devices like echocardiography and $\mathrm{x}$-ray for cardiac screening (Vukanovic-Criley JM, 2006).

To counter the subjectivity and the high percentage of diagnostic errors, computer-aided diagnostic (CAD) systems can provide paramount importance (Božo Tomas, 2007), (Belloni and Spoletini, 2007). For the successful implementation of CADs, the quality of the input signal should be high. Such automation has been researched for over six decades now. In the 1960s, one of the ground-breaking studies in the automatic classification of heart sound pathology was performed by (D. S. Gerbarg and Hofler, 1963). Since then thousands of research papers have been published. Some of the prominent works have been properly investigated in the report published in 2016 by (Liu C1, 2016). This paper demonstrates the importance of a well-characterized dataset for developing successful classification algorithms. This work also assembles the largest heart sound dataset.

The 2016 PhysioNet Computing in Cardiology Challenge was one of the most successful challenges conducted by the program which attracted a large number of researchers to solve the heart sound classification to normal and abnormal. In the competition, the largest heart sound dataset compiled by (Liu C1, 2016) was provided. The winners of the competition, Potes et al. (Cristhian Potes, 2016) have developed a deep-learning-based classifier that combines time-frequency features with a reported sensitivity of $96 \%$, specificity of $80 \%$ and overall accuracy of $89 \%$.

Almost all previously proposed algorithms needed the segmentation of the heart sound recording into first heart sound, second heart sound, systole, and diastole parts. This is a reasonable assumption which may lead to pinpointing of abnormalities in the heart sounds at specific temporal locations. However, the complexity and also the error introduced in the accurate localization of the segments have decreased the performance of the algorithms.

Recently, P. Langley and A. Murray (Cristhian Potes, 2017) have demonstrated the feasibility of accurate classification without segmentation of the heart sounds. The paper has a relatively lower overall accuracy of $79 \%$ (specificity $80 \%$, sensitivity $77 \%$ ) classification, and claims this is mainly due to the quality of the dataset used. Despite the sheer volume of research done in the area, the studies are critically hampered by the lack of high- quality recordings that have proper validation and standardization. This would have created common formatting that allows collaborative research, large- scale analytics, and tools and methodologies to be shared. The largest available open access data set is available which was compiled by Liu et al. (Liu C1, 2016). It contains 2435 heart sound recordings from 1297 subjects. The dataset consists of recordings from subjects with a variety of abnormalities which include heart valve damage and coronary artery disease. The maximum overall accuracy reported in the literature by using this database is only $94 \%$ which was achieved by introducing different model optimization techniques (Suhm, 2019).

D.B. Springer et al. (D.B. Springer, 2014) have worked on a dataset that is recorded to classify an RHD from normal heart sounds. A total of 318 recordings from 106 subjects where 40 were identified with RHD. Their aim was to detect systolic murmur hence the heart sound is segmented before feature extraction. A combination of MFCC and waveletfeatures are used. SVM classification algorithm is used by optimizing its parameters and the procedure is validated using a 10 -fold cross-validation technique. 
They reported a maximum $\mathrm{F} 1$ score of only 0.7 , the sensitivity of $74.8 \%$ and specificity of $74.5 \%$. Poor quality recording and external generator noise were to blame for such low performance. This demonstrates the necessity for a large and reliable dataset which takes into account specific pathology.

For the current study, we gathered one of the largest available heart sound data set by recording it from one hundred seventy subjects with a state-of-theart electronic stethoscope and addresses a particular type of valvular heart disease called RHD. Twentysix different features are used and the features are computed from a non-segmented data. These features include time-domain components, frequency components, and perceptual components. These features are extracted from the entire signal to properly deal with systolic as well as diastolic murmurs.

\section{MATERIALS AND METHODS}

\subsection{Data Collection}

The heart sound data was collected at Tikur Anbessa Referral Teaching Hospital, College of Health Sciences, Addis Ababa University, Addis Ababa, Ethiopia from August 2018 to July 2019. The study protocol was approved by the Research Ethics Committee of the Department of Internal Medicine (Ethical Clearance No: 014/2018).

The heart sound data were recorded from one hundred seventy subjects, one hundred twenty four were confirmed RHD patients (seventy four females, fifty males) with ages from 9 to 47 with mean and SD of $22.9 \pm 8.9$ years. The time since the first diagnosis is from two months to 20 years with mean and SD of $3.3 \pm 3.1$ years. Each diagnosis is confirmed by echocardiographic imaging and a cardiologist analysis. There were 46 normal subjects ( 15 females, 31 , males) with age 5 to 37 years with mean and SD of $14.4 \pm 10.5$ years. The electronic heart sound was recorded by ThinklabsOne ${ }^{\mathrm{TM}}$ digital stethoscope with a sampling frequency of $44.1 \mathrm{KHz}$. An average of 3.88 minutes of recording per subject is acquired. The digital stethoscope was positioned at the fifth intercostal space around the midclavicular line to properly record the mitral valve sound. The audio data is transferred to a mobile phone and is saved as a wav file.

\subsection{Visualization}

A ten-second waveform of a normal heart sound from a healthy subject is shown in Fig. 2 (top). In this fig- ure, the S1 and the S2 are clearly identifiable. Fig. 2 (bottom) shows the corresponding spectrogram to visualize how the energy is distributed over time. Clicks and glitches which are common features of a murmur can be very well visualized in the spectrogram.

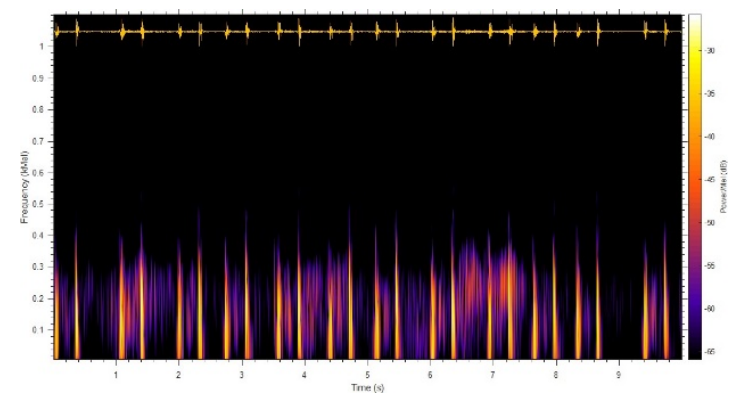

Figure 2: Healthy Subject: Time domain wave from (top) and spectrogram in Mel-frequency scale shown thermal colormap

Instead of a linear scale, the Mel- frequency scale is used to roughly resemble the resolution of the human auditory system. The spectrogram in the Melfrequency scale shows much of the spectral density that belongs to S1 and S2 whereas systole and diastole durations contain very little concentration. Fig. 3 shows is a recording from an RHD patient. In this recording, S1 and S2 can still be easily identified. However, looking at the corresponding spectrogram, it can be seen that there is a significant amount of energy in the systole and diastole parts which indicates the presence of murmur.

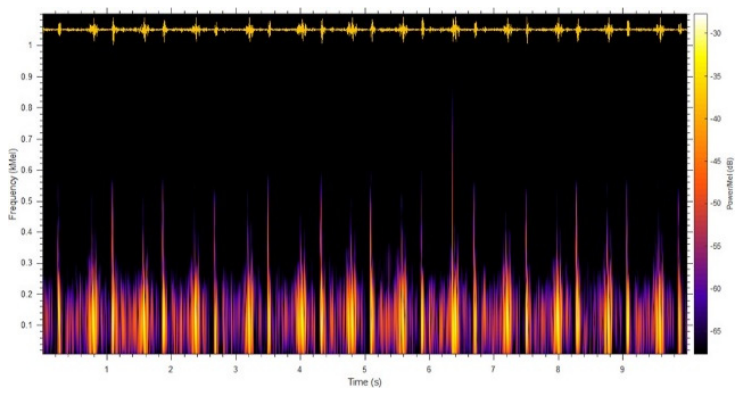

Figure 3: RHD Patient: Time domain wave from (top) and spectrogram in Mel-frequency scale shown thermal color map.

\subsection{System Architecture}

This section presents the complete workflow of developing a machine learning application to automatically detect RHD. The data acquisition, pre-processing, feature extraction, and classification steps are presented in detail. The classification performance of various classification algorithms is also investigated. 
The overall architecture of the system is shown in Fig. 4. Detail explanation of all the steps is presented below.

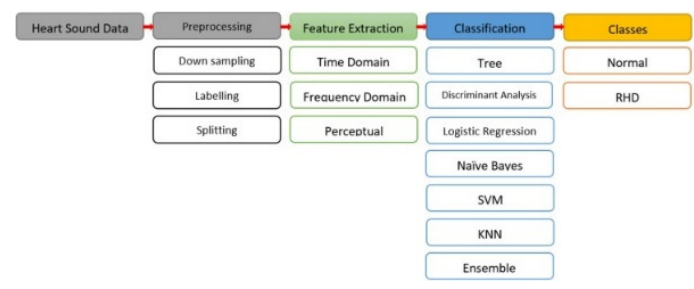

Figure 4: Overall System Architecture.

\subsubsection{Pre-processing}

The original data is sampled at $44.1 \mathrm{kHz}$. Experiments conducted at a sampling rate greater than or equal to $2 \mathrm{kHz}$ showed that the performance of the classification algorithm is not significantly affected. Hence, the data is down sampled to $2 \mathrm{kHz}$. Each record is labelled as Normal and RHD based on echocardiography analysis and cardiologist decision. The records are split to more manageable windows of 5 seconds and are ready for feature extraction. As the data is recorded in an uncontrolled environment and many of the recordings are corrupted by various types of noises such as movement artefact, talking, mobile phone interference, traffic sound, coughing, lung sounds, gastrointestinal sounds, pounding and clicks due to high volume recording. However, no filtering or noise removal was done on the data to make sure that the system resembles a real practice scenario.

\subsubsection{Feature Extraction}

In RHD patients, auscultation reveals the characteristic systolic murmur of mitral regurgitation. When the disease progresses, an additional diastolic murmur may also be present. The intensity of the murmur generally correlates with the severity of the disease (Liesl Zühlke, 2019). Different representations of the recorded heart sound are required to detect these abnormalities. In the literature, several features are proposed in order to properly characterize murmur due to RHD (Zümray Dokur, 2009). These features are extracted from the entire signal to properly deal with systolic as well as diastolic murmurs. Finally, twenty six features were extracted which include Time Domain Features (Median, Standard Deviation, Mean Absolution Deviation, 25 $5^{\text {th }}$ percentile, $75^{\text {th }}$ percentile, Inter Quartile Distance, Skewness, Kurtosis), Frequency Domain Features (Shannon's Energy, Spectral Entropy, Dominant Frequency, Energy Magnitude at the Dominant Frequency, Dominant Frequency Ratio), and Perceptual Features which first thirteen elements of the Mel frequency cepstral coefficients (MFCC). Table 1 presents the formulas and descriptions used in computing the above features.

\subsubsection{Classification}

There exist several machine learning algorithms specially tailored for predictive modeling. Selecting the best one is usually a tradeoff between speed of training, memory usage and predictive accuracy on new data. In medical applications, not only the accuracy but also specificity and sensitivity are also important. Several existing classification algorithms are experimentally compared to our heart sound dataset collected from one hundred seventy subjects. Support Vector Machines (SVM), K-Nearest Neighbor (KNN) classification algorithms have found to classify the data with superior accuracy.

\subsubsection{Validation}

The classification performance of the different algorithms is compared by the overall accuracy, sensitivity, specificity and F1-Score on the 30\% holdout validation data. The confusion matrix is also computed. Table 2 presents the validation parameters and corresponding formulas.

\section{RESULTS}

Two experiments are conducted to evaluate the classification performance of Cubic SVM and Fine KNN. The experimental results are shown in Table 3.

\subsection{Experiment I}

The first experiment is done on our collected heart sound data. This set has a total of 170 subjects (124 confirmed RHD patients and 46 Normal Subjects). A total of 3957 (2886 RHD and 1071 Normal) records with a 10 -second duration are used. This data is further split into 5-second intervals as mentioned in the pre-processing step. The data is recorded in an uncontrolled environment and many of the recordings are corrupted by various types of noises such as movement artefact, talking, mobile phone interference, traffic sound, coughing, lung sounds, gastrointestinal sounds, pounding and clicks due to high volume recording. No pre-processing or removal of the data was done to simulate an actual auscultation environment. For this setup, Cubic SVM has an over- 
Table 1: Formulas and description to compute features.

\begin{tabular}{|c|c|}
\hline Feature & Formula /Description \\
\hline Median & $\left(\frac{N+1}{2}\right)^{t h}$ \\
\hline Standard Deviation & $\sigma=\sqrt{\frac{1}{N-1} \sum_{i=1}^{N}\left|X_{i}-\mu\right|^{2}}$ \\
\hline $\begin{array}{l}\text { Mean Absolute } \\
\text { deviation }\end{array}$ & $\begin{array}{l}\text { mean.dev(x)= } \frac{1}{N} \sum_{i=1}^{N}\left|X_{i}-\mu\right| \\
\mathrm{X} \text { is the heart sound Signal }\end{array}$ \\
\hline Skewness & $s=\frac{E\left(X_{i}-\mu^{3}\right.}{\sigma^{3}}$ \\
\hline Kurtosis & $k=\frac{E\left|X_{i}-\mu\right|^{4}}{\sigma^{4}}$ \\
\hline Shannon Entropy & $H=\frac{-1}{N} \sum_{i=1}^{N} X_{i, \text { nom }}^{2} \log X_{i, \text { nom }}^{2}$ \\
\hline Spectral Entropy & $\begin{array}{l}H_{S}=-\sum_{m=1}^{N} \frac{P_{m} \log _{2} P_{m}}{\log _{2} N} \\
P_{m}=\frac{S}{\sum S_{i}} \cdot S=|f f t(X)|^{2}\end{array}$ \\
\hline $\begin{array}{l}\text { Dominant } \\
\text { Frequency }\end{array}$ & $\begin{array}{l}\text { The frequency at which the } \\
\text { maximum of the spectrum occurs }\end{array}$ \\
\hline $\begin{array}{l}\text { Dominant } \\
\text { Frequency ratio }\end{array}$ & $\begin{array}{l}\text { The ratio of the energy of the } \\
\text { maximum to the total energy of } \\
\text { the signal }\end{array}$ \\
\hline $\begin{array}{l}\text { Mel-frequency } \\
\text { Cepstral } \\
\text { Coefficients }\end{array}$ & $\begin{array}{l}\text { The steps followed are: } \\
\text { 1. preemphasise heart sound } \\
\text { signal using a first-order FIR filter } \\
\text { with preemphasis coefficient of } \\
\text { 0.97. } \\
\text { 2. Computer short-time Fourier } \\
\text { transforms. } \\
\text { 3. Magnitude spectrum com- } \\
\text { putation followed by filter bank } \\
\text { design with } 20 \text { triangular filters } \\
\text { uniformly spaced on the Mel scale } \\
\text { between } 10 \mathrm{~Hz} \text { and } 700 \mathrm{~Hz} \text { to span } \\
\text { the standard frequency range of } \\
\text { heart sounds. } \\
\text { 4. The filter bank is applied to } \\
\text { the magnitude spectrum values to } \\
\text { produce filter bank energies } \\
\text { (FBEs) (20 per frame) } \\
5 \text {. Log-compressed FBEs are } \\
\text { then decorrelated using the } \\
\text { discrete cosine transform to } \\
\text { produce cepstral coefficients. } \\
6 \text {. apply sinusoidal lifter to } \\
\text { produce liftered MFCCs. }\end{array}$ \\
\hline
\end{tabular}

all accuracy of $98.5 \%$ with $97.4 \%$ sensitivity, $99.0 \%$ specificity, and $98.1 \%$ precision. The corresponding PPV is $98 \%$ for normal and $99 \%$ for RHD.
Table 2: Validation Parameters.

\begin{tabular}{|l|c|}
\hline Parameter & Formula \\
\hline Accuracy & $T p+\frac{T p+T n}{T n+F p}+F n$ \\
\hline Sensitivity & $T \frac{T p}{p+F} p$ \\
\hline Specificity & $\frac{T n}{T n+F}$ \\
\hline Precision & $\frac{T p}{p+F} p$ \\
\hline
\end{tabular}

\subsection{Experiment II}

It is noted that both the training and validation sets are unbalanced. This can only demonstrate a clinical setup where there are more positives than negatives. This second experiment is done to check the robustness of the system against noise and different recording environments. For this purpose, 231 clean heart sound recordings from normal subjects and 120 heart sound recordings with a substantial amount of background noise and distortion are included from the Bentley et al. (Bentley et al., 2011) open dataset. These data are collected using iStethoscope ProTM iPhone app and DigiScopeTM from the general public and clinical trials. A total of 4308 (2886 RHD and 1422 Normal) records with a 10 -second duration is used for this experiment. The data is further split into a 5-second duration as mentioned in the preprocessing step. The Cubic SVM algorithm has again proved to be resilient to the noise by achieved $97.1 \%$ accuracy with $98 \%$ sensitivity, $95.3 \%$ of specificity and $97.6 \%$ precision. The corresponding PPV values are $96 \%$ for normal and $97 \%$ for RHD.

Table 3: Experimental Results.

\begin{tabular}{|c|c|c|c|c|c|c|c|}
\hline \multirow[b]{2}{*}{ Experiment } & \multirow[b]{2}{*}{ Algorithm } & \multicolumn{2}{|c|}{ Data Size } & \multirow{2}{*}{$\begin{array}{c}\text { Accuracy } \\
(\%)\end{array}$} & \multirow{2}{*}{\begin{tabular}{|c|} 
Sennsitivityl \\
$\%)$
\end{tabular}} & \multirow{2}{*}{$\begin{array}{c}\begin{array}{c}\text { Specificity( } \\
\%)\end{array} \\
\end{array}$} & \multirow{2}{*}{$\begin{array}{c}\begin{array}{c}\text { Precision } \\
(\%)\end{array} \\
\end{array}$} \\
\hline & & Normal & RHD & & & & \\
\hline \multirow{3}{*}{1} & CUBIC SVM & \multirow[b]{2}{*}{1071} & \multirow[b]{2}{*}{2886} & 98.5 & \begin{tabular}{|r|}
97.4 \\
\end{tabular} & 99 & $\begin{array}{r}98.1 \\
\end{array}$ \\
\hline & FINE KNN & & & 98 & 97.5 & 98.2 & 96.4 \\
\hline & CUBIC SVM & \multirow[b]{2}{*}{1422} & \multirow[b]{2}{*}{2886} & 91.1 & 98 & 95.3 & 97.5 \\
\hline ॥ & FINE KNN & & & 96.7 & 97.3 & 95.7 & 97.6 \\
\hline
\end{tabular}

\section{DISCUSSION AND CONCLUSIONS}

Although heart sounds can actually tell you a lot about a patient, auscultation proficiency is declining fast. This is mainly due to the intrinsic weakness of the human auditory system and the technical ability gap in which a clinician can explain why I need to know it where there is more advanced technology to do it. Due to this instead of auscultation, echocardiography is considered as a gold standard to screen and diagnose valve damage in the heart. Unfortunately, in developing countries, such an advanced device is very expensive and cardiologists who can use it are rare. 
To make matters worse, the burden of cardio-vascular disease due to the non-curable but treatable and preventable rheumatic heart disease is very high in these countries. The damage to the heart valves due to RHD can be reduced if it is detected early. The most straightforward and cheapest approach to detect valvular damage is detected by listening to the heart murmurs. In this study, one of the largest heart sound dataset is collected. Using this dataset, a comprehensive machine learning method is deployed. This study has demonstrated the performance of the machine learning algorithm with extensive characterization methods to quantify and accurately classify heart sounds of a normal and confirmed Rheumatic Heart Disease. A total of 26 features that encompass time domain, frequency domain, and perceptual characteristics are carefully selected and computed. The results of the current study are very promising with respect to classification accuracy of $97.1 \%$, with $98 \%$ sensitivity, $95.3 \%$ specificity, and $97.6 \%$ precision and a positive predictive value of $99 \%$ in detecting RHD. Our data depicts better results in terms of classification accuracy, sensitivity, and specificity than previously reported studies available on heart sound data. While this study considers a specific type of heart disease, the other studies were trying to model generic types of heart diseases.

Furthermore, we hope that this technology coupled with mobile phone devices can be used as a screening tool in a clinical environment where access to echocardiography and cardiologist is difficult. This will make it a timely, affordable and reliable detection tool allowing a non-medically trained individual to diagnose and screen for RHD.

\section{REFERENCES}

Belloni, D. Della Giustina, S. R. M. R. and Spoletini, E. (2007). Towards a computer-aided diagnosis by means of phonocardiogram signals", industrial elec- tronics. IEEE International Symposium.

Bentley, P., Nordehn, G., Coimbra, M., and Mannor, S. (2011). The pascal classifying heart sound challenge 2011. CHSC2011.

Božo Tomas, D. Z. (2007). Heart sound lines - proposal of a novel heart auscultation assistant diagnosis tool,. International Journal of Latest Trends in Engineering and Technology.

Cristhian Potes, Saman Parvaneh, A. R. B. C. (2016). Ensemble of feature-based and deep learning-based classifiers for detection of abnormal heart sounds. Computing in Cardiology.

Cristhian Potes, Saman Parvaneh, A. R. B. C. (2017). Heart sound classification from unsegmented phonocardiograms. Physiol. Meas.
D. S. Gerbarg, A. Taranta, M. S. and Hofler, J. J. (1963). Computer analysis of phonocardiograms. Progress in Cardiovascular Diseases.

D.B. Springer, L. Z. B. M. L. T. G. C. (2014). Mobile phone-based rheumatic heart disease diagnosis. IEEE Conference on Appropriate Healthcare Technologies for Low Resource Settings.

Emelia J. Benjamin, Paul Muntner, A. A. M. S. B.-C. W. C. A. P. C. A. M. C. (2019). Heart disease and stroke statistics-2019 update: A report from the american heart association, volume 139, no. 10. Circulation.

Liesl Zühlke, F. P. (2019). Clinical manifestations and diagnosis of rheumatic heart disease. UpToDate.

Liu C1, Springer D, L. Q. M. B. J. R.-C. F. C. F. (2016). An open-access database for the evaluation of heart sound algorithm. Physiol Meas.

Pelech, N. (2004). The physiology of cardiac auscultation. Pediatric Clinic North America.

Suhm, B. (2019). Heart sound classifier. MATLAB Central File Exchange.

Vukanovic-Criley JM, Criley S, W. C. B. J. G.-M. L. C. W. N. W. C. J. (2006). Competency in cardiac examination skills in medical students, trainees, physicians, and faculty: a multicenter study. Arch Intern Med.

Walsh, J. C. A. B. G. M. W. (2019). In The Australian guideline for prevention, diagnosis, and management of acute rheumatic fever and rheumatic heart disease. Australia RHD.

Watkins DA, Johnson CO, C. S. K. G. B. A.-B. G. (2017). Global, regional, and national burden of rheumatic heart disease, 1990-2015. New England Journal of Medicine.

WHO Annual Report (2013). WHO global action plan for the prevention and control of noncommunicable diseases 2013-2020. WHO.

Zümray Dokur, T. (2009). Feature determination for heart sounds based on divergence analysis. Elsevier, Digital Signal Processing. 